For example, silica in any form is commonly regarded as being completely insoluble in water at ordinary temperatures. Under low pressures of liquid water a slight solubility can be shown, but this drops almost to zero as the critical temperature is approached. Under pressures of the order of 1,000 atmospheres of water vapour, however, a different state of affairs is found, for with increasing temperature the solubility progressively increases and has been determined up to a value of about $\frac{1}{3}$ per cent. These observations are clearly relevant to some problems of silica transport.

Unquestionably the most important family of minerals in igneous and metamorphic rocks is the feldspar family. Microscopic, heat treatment and X-ray studies have shown the group to be complex. Dry-melt investigation of feldspar crystallization has been laborious because the alkali feldspars take weeks or months to crystallize; but, by contrast, crystallization is completed within an hour in the presence of water vapour at a pressure of one thousand atmospheres. Recent high-pressure work has confirmed the existence of a high-temperature series and of a low-temperature series of plagioclase feldspars, with the consequence that a number of difficulties attending the identification of feldspars in lavas and porphyries are well on the way towards being cleared up. A further most important conclusion is that the stability relations observed between the alkali feldspars and leucite under dry-melt conditions broadly apply in the presence of water vapour under pressure, crystallization temperatures being much reduced to figures quite compatible with our current notions of magmatic temperatures. The value of earlier dry. melt work is thus confirmed.

A further notable research is that on the system $\mathrm{MgO}-\mathrm{SiO}_{2}-\mathrm{H}_{2} \mathrm{O}$. The temperature/pressure stability curves for substances such as pure forsterite, olivine, serpentine, enstatite, talc and periclase in the presence of water have been determined. The conditions under which serpentinization may and may not take place in Nature have been indicated, and the conclusion has been drawn that neither a dunite magma nor a serpentine magma is likely ever to exist. A possible mechanism for the formation of dyke-like masses of olivine rock in pyroxenites, depending on silica transport by water vapour, has been outlined.

The future. That hydrothermal research will play an increasing and vital part in formulating petrogenetic theory seems certain.

The keystone of further progress must be the study of the hydrostatic phase equilibria. Certain problems, such as the progressive decarbonation of a siliceous dolomite, discussed by Bowen and Tilley, offer a prospect of unusual interest and elegance. Replacement phenomena will continue to be enigmatic until the relevant phase-equilibrium conditions have been determined.

A topic of great interest concerns the primary or secondary nature of the water so copiously evolved as steam during volcanic eruption, and is a reminder that our knowledge of the solubility of water in molten silicates is fragmentary. Goranson's work on granite melts, twenty years ago, requires extension to melts prepared from other igneous rock types. The carbonatites present an intriguing puzzle in this connexion.

Quantitative assessment is yet barely possible of the magnitude of the forces responsible for the penetrative shearing movement and preferred orientation demonstrated in some metamorphic fabrics. This problem is a challenge to experimental ingenuity and, in addition, calls for a study of the kinetics of mineral crystal disintegration and growth at the atomic level.

Large-scale diffusion has been put forward repeatedly as an important petrogenetic process by many petrologists. There is no branch of the subject where quantitative laboratory evidence is so scant, and where there is greater need for a strict physicochemical treatment of the problem.

\section{CHEMOTHERAPY OF TUBERCULOSIS}

A COLLOQUTUM on the chemotherapy of tuberculosis was held in Dublin during July 10-13. It was sponsored by the Medical Research Council of Ireland, and the organization was in the hands of a small committee under the chairmanship of Dr. V. C. Barry. The meeting was international in character, the speakers being drawn from Great Britain, the United States, Germany, France and Ireland. There was a registered membership of 170 , more than seventy of whom were overseas visitors, representing as well as the above countries, Switzerland, Holland and Sweden. The members came together for the first time on the eve of the opening at a reception given in Newman House by the President of University College and Mrs. Tierney.

The official opening of the colloquium in University College on July 10 was by the Minister for Health and Social Welfare, Dr. James Ryan. Prof. R. P. Farnan, chairman of the Medical Research Council, presided, and the attendance included the Taoiseach, Mr. de Valera. The colloquium was divided into seven sessions, each of which occupied a morning or afternoon. On Thursday afternoon the members had the honour of being received by the President of Ireland and Mrs. O'Kelly at Áras an Uachtaráin.

Each session was opened by a lecture and, after an interval for light refreshments, a discussion lasting about an hour and a half followed. The most interesting feature of the colloquium was that it brought together for four days the organic chemist and the biochemist, the bacteriologist, the pharmacologist and the clinician. The arrangement of the lectures was a logical one, beginning with the chemistry and biochemistry of the tubercle bacillus, followed by the organic chemists' approach to the problem of producing an adequate chemotherapeutic agent for tuberculosis. The story was continued with a description of the methods of preclinical evaluation of the agent. In the fourth lecture the present status of antibiotics in tuberculosis was assessed. At this stage the clinicians took over, and the last three lectures dealt with the thiosemicarbazones in tuberculosis, and the clinical evaluation of potential chemotherapeutic agents in pulmonary and urogenital tuberculosis. The formal and informal discussions which arose in this heterogeneous scientific gathering must prove of great value to all workers in this field.

Prof. Jessop presided at the first lecture, which was delivered by Dr. E. Lederer, on the chemistry and biochemistry of mycobacteria. This was an extensive review of the literature since 1940 on the carbohydrates, proteins and lipids of the bacilli. Dr. Lederer emphasized the extreme variability of the composition of mycobacteria and showed how it 
depends on environmental factors and the age of the culture. Perhaps of greatest interest was his account of the researches of his colleagues and himself on the mycolic acids. Their probable formula is $\mathrm{C}_{88} \mathrm{H}_{178} \mathrm{O}_{4}$, which contains a- $-\mathrm{COOH}$, an $-\mathrm{OH}$ and an $-\mathrm{OCH}_{3}$ group. Pyrolysis of the mycolic acid at $300^{\circ}$ in vacuo gives $n$-hexacosanoic acid, $\mathrm{C}_{26} \mathrm{H}_{52} \mathrm{O}_{2}$; chromic acid oxidation yields among other products stearic acid, $n$-hexacosanoic acid, hexadecane and a 1 : 16-dicarboxylic acid. Chromatographic purification on alumina has led to the isolation of two distinct, and apparently isomeric, mycolic acids $(\alpha$-and $\beta$-). Degradation experiments indicate that a mycolic acid is a high molecular weight $\beta$-hydroxy acid with a long aliphatic side-chain in the $\alpha$-position $(\alpha-n$ tetracosyl- $\beta$-hydroxy acid). The difference between the $\alpha$-and $\beta$-acids must reside in the $C_{62}$ fragment, which still awaits investigation. Human virulent strains of Myco-tuberculosis contain methoxylated mycolic acids, while the H37 RA avirulent strain contains an $\alpha$-mycolic acid free from methoxyl. The interesting new amino-acid, $\alpha, \varepsilon$-diamino-pimelic acid, which Work isolated for the. first time from the diphtheria bacillus, has now been found in all strains of Myco. bacteria so far examined. Among other matters of interest covered in the lecture was the problem of acid-fastness. It seems that all our present knowledge of this phenomenon can be explained by the observation of Yegian and Vanderlinde, who have shown that, in the stained bacterial cell, the greater part of the dye is in solution inside the cell. Its introduction is effected by heating with a phenol solution of fuchsin, and it cannot be removed by acid-alcohol washing since the cell wall is impermeable to this solvent. Boiling alcohol does remove the stain. Acid-fastness is probably due, therefore, to the presence of a characteristic mycolic acid containing lipopolysaccharide in the cell membrane.

During the discussion which followed, Dr. C. Iland referred to work carried out with Prof. M. Stacey's team at Birmingham. A urea extract of young living tubercle bacilli gives a complex material which has the biological properties of fresh unheated tuberculin. It yields an electrophoretically homogeneous protein which gives a complement fixation test with human tuberculous sera. The use of urea for extracting living cells may prove of particular value in providing cheaply a tuberculin skin-testing reagent. Prof. R. A. Q. O'Meara pointed out that the subject of Dr. Lederer's address was the end-products of mycobacterial metabolism; of greater importance from the point of view of chemotherapy are the products of the intermediate phases of metabolism, the substances which are most elusive are likely to be the most important. Dr. D. Twomey referred to the isolation of branched-chain fatty acids from other natural sources; for example, the iso- and anteisoseries of acids isolated from wool-fat, and phytomonic acid from $P$. tumefaciens. These latter acids probably exist as such in Nature, unlike phthioic acid, which is now known to be an artefact. Dr. Twomey also provoked a discussion on 'permeability', which he suggested exists as a crude conception in the minds of many chemists and biologists.

In the second lecture, Dr. V. C. Barry described an organic chemist's approach to the chemotherapy of tuberculosis. He reviewed the work of his team, which is a unit of the Medical Research Council of Ireland. They have produced some drugs of potential -value in tuberculosis and leprosy. B.53, a dialkyl succinic acid half-ester, has been used successfully in the treatment of tuberculosis of the urinary bladder. This substance has low in vitro activity in the presence of serum. A planned synthetical approach led to the production of a series of long branchedchain hydroxyamines which are much less antagonized by serum. One of these, $B .390$, a branched-chain pentadecylamine derivative, has shown a suppressive effect in guinea pig tuberculosis when given orally. A series of long-chain alkylpyrrolidines and piperidines was also described. In Dr. Barry's experience, this was the first example of a fatty compound exhibiting anti-tubercular activity in vivo, and it provides some justification for the long preoccupation of workers in this field with substances of a fatty nature. Another aspect of the work showed the development from the lichen product, diploicin, by successive synthetical modifications, of a series of phenazine dyes highly active against the bacillus in serum broth. One of these, B.283 (2-anilino-3-imino-5phenyl-phenazine), has shown a suppressive effect in mouse and guinea pig tuberculosis. Dr. Barry described six types of compound which up to date are known to modify the disease in the guinea pig. These were all produced empirically. The testing of antitubercular compounds in liquid media enriched by serum can be relied on to make possible the selection of agents likely to exhibit activity in vivo. Dr. J. Barnes exhibited a series of photographs showing the effect of $B .283$ in a series of cases of lepromatous leprosy in Nigeria. In his view, $B .283$ is more rapidly effective than any other drug in current use for the treatment of this form of leprosy. A very lively discussion followed to which Dr. D. A. Peak, Mr. F. A. Robinson, Prof. J. Algar, Dr. Lederer and others contributed. The discussion centred for the most part on the question of the empirical versus the rational approach to chemotherapy. It was remarkable for the high level of honesty sustained, there being general agreement that no successful chemotherapeutic agent has yet been discovered by a completely rational approach. Prof. T. S. Wheeler presided at this session.

Dr. W. H. Feldman spoke on the preclinical appraisal of antituberculosis agents. He emphasized that certain prerequisites must be satisfied before a given agent is considered worthy of clinical trial. This necessitates the collection of a considerable amount of data from many experimental studies. He discussed in detail in vitro testing, the in vivo-in vitro test and the conduct of many in vivo screening routines. These included the use of the chick embryo, the baby chick, the rabbit (intracorneal infection), the mouse, the hamster and the guinea pig. No summary of this lecture is possible. The refinement and careful control of Dr. Feldman's in vivo experiments are wholly admirable. However, animal experimentation is not an end in itself, and in the present writer's view there is a stage in its development when further elaboration may lead only to results of purely theoretical significance. In the discussion, Mr. Francis compared the pathology of the disease in the monkey, dog and smaller animals. The dog does not develop much allergy, and must be regarded as a resistant animal. Dr. J. Ungar expressed the belief that in vitro testing is valueless unless carried out in complex media rich in protein. Substances with marked reticulocytic stimulation influence mice more than guinea pigs. The effect of streptomycin in experimental tuberculosis in the mouse is quite different from that in the guinea pig. Further contributors to the discussion were Dr. 
A. Q. Wells, Dr. Carmichael, Dr. Conalty and Mr. W. Freeman. Prof. F. S. Stewart presided.

In the fourth session, the chair was occupied by Prof. T. G. Moorhead. Dr. George Brownlee opened the proceedings with a talk on the present status of antibiotics in tuberculosis. This was a stimulating and original contribution, which characteristically had little relevance to the title. Dr. Brownlee described six biochemical lesions which follow invasion by a tubercle bacillus : multiplication of the parasite, chemiotaxis of monocytes, inhibition of proteases, hypersensitivity effect, skin allergy and acquired resistance. Specific antimicrobial chemotherapy is associated only with the first. Three factors influence the effect of chemotherapeutic agents on a bacterial population : the efficiency of the agent, the effect of the number of the organisms, and the rate of emergence of resistant strains. Dr. Brownlee dealt at length with the role of hypersensitivity in the spread of the disease. He visualized the advent of an anti-anaphylactic drug which will effectively modify tuberculin hypersensitivity. This may be expected to revolutionize our present-day concepts of the limitations of conventional chemotherapy of tuberculosis. $\mathrm{H}_{\theta}$ also expressed the opinion that the evidence appears to be complete that a chemical entity exists in suitably prepared dead tuberclo bacilli, which is antigenic and protective. Though this substance may be labile in character, he believed that ways will be found of extracting it. Opening the discussion, Dr. D. G. Madigan described the treatment of a group of patients with bacillary emulsion under the cover of effective chemotherapy with streptomycin and $p$-aminosalicylic acid. In 50 per cent of patients, it is possible to achieve desensitization as measured by the skin test (to 1 in 10) in six weeks. Dr. P. Swift described the treatment of tuberculous meningitis with streptomycin. In his opinion it is not now justifiable to use streptomycin by itself. The remainder of the session was devoted to a lively discussion on the origin and nature of streptomycin resistance, in which Prof. Kennedy, Dr. D. A. Mitchison, Mr. G. W. Dawson, Dr. J. Crofton and Prof. Julius took part.

The second half of the colloquium opened in Trinity College with Dr. Gerhard Domagk's lecture, Prof. W. R. Fearon presiding. Prof. Domagk said that three compounds have been used in the treatment of chronic tuberculosis infection : the thiosemicarbazones (conteben), streptomycin and $p$-aminosalicylic acid. All three are active against different types of tubercle bacilli both in vitro and in vivo. In the experimental animal, a combination of conteben and streptomycin has proved more effective than streptomycin alone or any other combination. Side reactions at first attributed to the thiosemicarbazone itself are to some extent due to dead or damaged bacilli. It is possible to obtain extensive fatty degeneration of the liver by injection of dead bacilli only. The thiosemicarbazones are being used extensively in South America and Central Africa for the treatment of leprosy. In the discussion, Dr. A. R. Martin pointed out that $T B$ III ( $p$-ethylsulphonylbenzaldehyde thiosemicarbazone) is better tolerated in man than conteben and produces much higher blood-levels with equal dosage. Dr. Barnes described a trial which he has conducted with a series of cases of tuberculoid and lepromatous leprosy. Conteben was effective in the former type, but very much less effective with the latter. In reply to Mr. Francis, whose experience has been that none of the partially successful tuberculosis and leprosy drugs have any appreciable effect on rat leprosy, Dr. Domagk pointed out that the strain of leprosy bacillus infecting man is quite different from the rat leprosy bacillus. It also emerged from the discussion that there is little information available as to the effect of thiosemicarbazones on streptomycin-resistant strains of tubercle bacilli.

The sixth and seventh lectures were mainly of interest to clinicians. Dr. Marc Daniels spoke on "The Clinical Evaluation of Potential Chemotherapeutic Agents in Pulmonary Tuberculosis". $\mathrm{He}$ described the various rigorous procedures and teamwork which alone can make a clinical trial of a new drug of value. Prof. J. M. O'Connor presided.

At the final session, Prof. J. McGrath presiding, Mr. J. T. D. Lane described his experience in the treatment of a number of cases of renal tuberculosis with streptomycin and a smaller number with B.283. The latter cases had failed to respond to strepto mycin. It was clear that both drugs are more effective when dealing with early lesions. He thought. it likely that a combination of the two drugs may prove more effective than either alone. In vesical tuberculosis persisting after nephrectomy, B.53. appears to be very effective. In this type of case, in his experience, streptomycin is only effective when the lesions are superficial.

It was a highly successful and lively conference, owing much of its success to the presence of workers interested in every stage of the chemotherapy of the disease, to the logical arrangement of the programme and finally to the fact that the limited number of contributions did not produce the mental indigestion frequently associated with gatherings of this kind. 'The chemists' contributions were tinged somewhat with melancholy, but not completely despairing. The biologists were optimistic and fruitful in theories. The bacteriologists among them appeared to be quietly confident that their routine procedures were adequate to any demands that might be made on them. The clinicians, however, as instanced by Dr. E. T. Freeman, were polite but firm in pointing out that the desired chemotherapeutic agent for tuberculosis has yet to be discovered.

A complete record of the proceedings will be published later this year as a special issue of the Irish Journal of Medical Science. VINCENT C. BARRY

\section{THE FAWLEY REFINERY}

$F^{\circ}$ OR the past two years, ever since the first grader started site-clearance in July 1949, the word 'Fawley' has taken on a new meaning in British oil circles. The place itself was of small consequence on a comparatively little-known peninsula between Beaulieu River and Southampton Water, save for the existence of the original Esso Petroleum Company's oil refinery, in operation for some thirty years past. All this has now changed. The new Esso refinery, officially opened by the Prime Minister on September 14, is an accomplished fact, a triumph of American and British brains and skill, a monument to the close ties binding the two countries in peace as well as in war.

The history of this outstanding example of initiative and enterprise is as impressive as is the vista of the whole complex plant itself. Begun in 1949, it was 